\title{
Design of Fuzzy Set-Based Polynomial Neural Networks with the Aid of Symbolic Encoding and Information Granulation
}

\author{
Sung-Kwun Oh, In-Tae Lee, and Hyun-Ki Kim \\ Department of Electrical Engineering, The University of Suwon, \\ San 2-2 Wau-ri, Bongdam-eup, Hwaseong-si, Gyeonggi-do, 445-743, South Korea \\ ohsk@suwon.ac.kr
}

\begin{abstract}
In this paper, we introduce fuzzy-neural networks- Fuzzy Polynomial Neural Networks (FPNN) with fuzzy set-based polynomial neurons (FSPN) whose fuzzy rules include the information granules (about the real system) obtained through Information Granulation. We have developed a design methodology (genetic optimization using Genetic Algorithms) to find the optimal structure for fuzzy-neural networks that expanded from Group Method of Data Handling (GMDH). It is the number of input variables, the order of the polynomial, the number of membership functions, and a collection of the specific subset of input variables that are the parameters of FPNN fixed by aid of genetic optimization that has search capability to find the optimal solution on the solution space. We adopt fuzzy set-based fuzzy rules as substitute for fuzzy relation-based fuzzy rules and apply the concept of Information Granulation to the proposed fuzzy set-based rules. The performance of genetically optimized FPNN (gFPNN) with fuzzy set-based polynomial neurons (FSPN) composed of fuzzy set-based rules is quantified through experimentation where we use a number of modeling benchmarks data which are already experimented with in fuzzy or neurofuzzy modeling.
\end{abstract}

\section{Introduction}

It is expected that efficient modeling techniques should allow for a selection of pertinent variables and a formation of highly representative datasets. Furthermore, the resulting models should be able to take advantage of the existing domain knowledge (such as a prior experience of human observers or operators) and augment it by available numeric data to form a coherent data-knowledge modeling entity. Most recently, the omnipresent trends in system modeling are concerned with a broad range of techniques of computational intelligence(CI) that dwell on the paradigm of fuzzy modeling, neurocomputing, and genetic optimization[1, 2]. The list of evident landmarks in the area of fuzzy and neurofuzzy modeling [3,4] is impressive. While the accomplishments are profound, there are still a number of open issues regarding structure problems of the models along with their comprehensive development and testing.

As one of the representative advanced design approaches comes a family of selforganizing networks with fuzzy polynomial neuron (FPN) (called "FPNN" as a new 
category of neuro-fuzzy networks) $[5,8,12]$. The design procedure of the FPNNs exhibits some tendency to produce overly complex networks as well as comes with a repetitive computation load caused by the trial and error method being a part of the development process.

In this paper, in considering the above problems coming with the conventional FPNN $[9,8,12]$, we introduce a new structure of fuzzy rules as well as a new genetic design approach. The new structure of fuzzy rules based on the fuzzy set-based approach changes the viewpoint of input space division. In other hand, from a point of view of a new understanding of fuzzy rules, information granules seem to melt into the fuzzy rules respectively. The determination of the optimal values of the parameters available within an individual FSPN leads to a structurally and parametrically optimized network through the genetic approach.

\section{The Architecture and Development of FSPNN}

The Fuzzy Set-based Polynomial Neuron(FSPN) encapsulates a family of nonlinear "if-then" rules. When put together, FSPNs results in a self-organizing Fuzzy Setbased Polynomial Neural Networks (FSPNN). Each rule reads in the form.

$$
\begin{aligned}
& \text { if } x_{p} \text { is } \boldsymbol{A}_{\boldsymbol{k}} \text { then } z \text { is } P_{p k}\left(x_{i}, x_{j}, \boldsymbol{a}_{p k}\right) \\
& \text { if } x_{q} \text { is } \boldsymbol{B}_{\boldsymbol{k}} \text { then } z \text { is } P_{q k}\left(x_{i}, x_{j}, \boldsymbol{a}_{q k}\right)
\end{aligned}
$$

where $\boldsymbol{a}_{q k}$ is a vector of the parameters of the conclusion part of the rule while $P\left(x_{i}, x_{j}\right.$, a) denoted the regression polynomial forming the consequence part of the fuzzy rule. The activation levels of the rules contribute to the output of the FSPN being computed as a weighted average of the individual condition parts (functional transformations) $P_{K}$. (note that the index of the rule, namely " $k$ " is a shorthand notation for the two indices of fuzzy sets used in the rule (1), that is $K=(l, k))$.

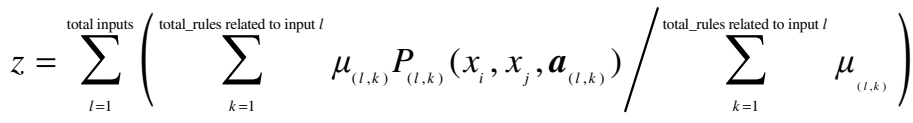

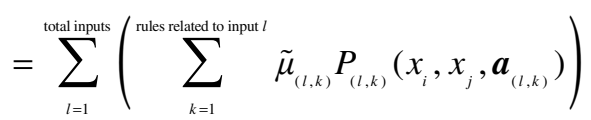

In the above expression, we use an abbreviated notation to describe an activation level of the " $k$ "th rule to be in the form

$$
\tilde{\mu}_{(l, k)}=\frac{\mu_{(l, k)}}{\sum_{k=1}^{\text {total rule related to input } l} \mu_{(l, k)}}
$$

When developing an FSPN, we use genetic algorithms to produce the optimized network. This is realized by selecting such parameters as the number of input variables, the order of polynomial, and choosing a specific subset of input variables. Based on the genetically optimized number of the nodes (input variables) and the polynomial order, refer to Table 1, we construct the optimized self-organizing network architectures of the FSPNNs. 
Table 1. Different forms of the regression polynomials forming the consequence part of the fuzzy rules

\begin{tabular}{|c||c||c|c||}
\hline $\begin{array}{l}\text { Order of } \\
\text { the polynomial }\end{array}$ & 1 & 2 & 3 \\
\hline \hline 0 (Type 1) & Constant & Constant & Constant \\
\hline 1 (Type 2) & Linear & Bilinear & Trilinear \\
\hline 2 (Type 3) & Quadratic & Biquadratic-1 & Triquadratic-1 \\
\hline 2 (Type 4) & Biquadratic-2 & Triquadratic-2 \\
\hline
\end{tabular}

1: Basic type, 2: Modified type

\section{Information Granulation Through Hard C-Means Clustering Algorithm}

We assume that given a set of data $X=\left\{\mathbf{x}_{1}, \mathbf{x}_{2}, \ldots, \mathbf{x}_{n}\right\}$ related to a certain application, there are some clusters which are capable of being found through HCM. The center point and the membership elements represent each cluster. The set of membership elements is crisp. To construct a fuzzy mode, we should transform the crisp set into the fuzzy set. The center point means the apex of the membership function of the fuzzy set. Let us consider building the consequent part of fuzzy rule. We can think of each cluster as a sub-model composing the overall system. The fuzzy rules of Information Granulation-based FSPN are as followings.

$$
\begin{aligned}
& \text { if } x_{p} \text { is } \boldsymbol{A}^{*}{ }_{k} \text { then } z-m_{p k}=P_{p k}\left(\left(x_{i}-v_{p k}^{i}\right),\left(x_{j^{-}} v_{p k}^{j}\right), a_{p k}\right) \\
& \text { if } x_{q} \text { is } \boldsymbol{B}^{*}{ }_{k} \text { then } z-m_{q k}=P_{q k}\left(\left(x_{i}-v_{q k}^{i}\right),\left(x_{j}-v_{q k}^{j}\right), a_{q k}\right)
\end{aligned}
$$

Where, $\boldsymbol{A}^{*}{ }_{\boldsymbol{k}}$ and $\boldsymbol{B}^{*}{ }_{\boldsymbol{k}}$ mean the fuzzy set, the apex of which is defined as the center point of information granule (cluster) and $m_{p k}$ is the center point related to the output variable on cluster ${ }_{p k}, v_{p k}^{i}$ is the center point related to the $i$-th input variable on clus$\operatorname{ter}_{p k}$ and $\boldsymbol{a}_{q k}$ is a vector of the parameters of the conclusion part of the rule while $P\left(\left(x_{i^{-}}\right.\right.$ $\left.\left.v^{i}\right),\left(x_{j^{-}} v^{j}\right), a\right)$ denoted the regression polynomial forming the consequence part of the fuzzy rule which uses several types of high-order polynomials (linear, quadratic, and modified quadratic) besides the constant function forming the simplest version of the consequence; refer to Table 1 . If we are given $m$ inputs and one output system and the consequent part of fuzzy rules is linear, the overall procedure of modification of the generic fuzzy rules is as followings. The given inputs are $X=\left[\mathbf{x}_{1} \mathbf{x}_{2} \ldots \mathbf{x}_{\mathrm{m}}\right]$ related to a certain application, where $\mathbf{x}_{\mathrm{k}}=\left[\mathrm{x}_{\mathrm{k} 1} \ldots \mathrm{x}_{\mathrm{kn}}\right]^{\mathrm{T}}, \mathrm{n}$ is the number of data and $\mathrm{m}$ is the number of variables and the output is $\mathbf{Y}=\left[\begin{array}{llll}\mathrm{y}_{1} & \mathrm{y}_{2} & \ldots & \mathrm{y}_{\mathrm{n}}\end{array}\right]^{\mathrm{T}}$.

Step 1) build the universe set

Universe set $\mathrm{U}=\left\{\left\{x_{11}, x_{12}, \ldots, x_{1 m}, y_{1}\right\},\left\{x_{21}, x_{22}, \ldots, x_{2 m}, y_{2}\right\}, \ldots,\left\{x_{n 1}, x_{n 2}, \ldots, x_{n m}, y_{n}\right\}\right\}$

Step 2) build $m$ reference data pairs composed of $\left[\mathbf{x}_{1} ; \mathrm{Y}\right],\left[\mathbf{x}_{2} ; \mathrm{Y}\right]$, and $\left[\mathbf{x}_{\mathrm{m}} ; \mathrm{Y}\right]$.

Step 3) classify the universe set $U$ into $l$ clusters such as $c_{i 1}, c_{i 2}, \ldots, c_{i 1}$ (subsets) by using HCM according to the reference data pair $\left[\mathbf{x}_{\mathrm{i}} ; \mathrm{Y}\right]$. Where $\mathrm{c}_{i j}$ means the $j$-th cluster (subset) according to the reference data pair $\left[\mathbf{x}_{\mathrm{i}} ; \mathrm{Y}\right]$. 
Step 4) construct the premise part of the fuzzy rules related to the $i$-th input variable $\left(\mathrm{x}_{\mathrm{i}}\right)$ using the directly obtained center points from HCM.

Step 5) construct the consequent part of the fuzzy rules related to the $i$-th input variable $\left(\mathrm{x}_{\mathrm{i}}\right)$. On this step, we need the center points related to all input variables. We should obtain the other center points through the indirect method as followings.

Sub-step1) make a matrix as equation (5) according to the clustered subsets

$$
A_{j}^{i}=\left[\begin{array}{cccc:c}
x_{21} & x_{22} & \cdots & x_{2 m} & y_{2} \\
x_{51} & x_{52} & \cdots & x_{5 m} & y_{5} \\
x_{k 1} & x_{k 2} & \cdots & x_{k m} & y_{k} \\
\vdots & \vdots & \cdots & \vdots & \vdots
\end{array}\right]
$$

Where, $\left\{x_{k l}, x_{k 2}, \ldots, x_{k m}, y_{k}\right\} \in \mathrm{c}_{\mathrm{ij}}$ and $\mathrm{A}_{j}^{i}$ means the membership matrix of $\boldsymbol{j}$-th subset related to the $\boldsymbol{i}$-th input variable.

Sub-step2) take an arithmetic mean of each column on $\mathrm{A}_{j}^{i}$. The mean of each column is the additional center point of subset $c_{i j}$. The arithmetic means of column is equation (6)

$$
\text { center point }=\left[\begin{array}{llll:l}
v_{i j}^{1} & v_{i j}^{2} & \cdots & v_{i j}^{m} & m_{i j}
\end{array}\right]
$$

Step 6) if $\mathrm{i}$ is $\mathrm{m}$ then terminate, otherwise, set $\mathrm{i}=\mathrm{i}+1$ and return step 3.

\section{The Design Procedure of Genetically Optimized FSPNN}

The framework of the design procedure of the genetically optimized Fuzzy Polynomial Neural Networks (FPNN) with fuzzy set-based PNs (FSPN) comprises the following steps

[Step 1] Determine system's input variables

[Step 2] Form training and testing data

[Step 3] specify initial design parameters

[Step 4] Decide upon the FSPN structure through the use of the genetic design

[Step 5] Carry out fuzzy-set based fuzzy inference and coefficient parameters estimation for fuzzy identification in the selected node(FSPN)

[Step 6] Select nodes (FSPNs) with the best predictive capability and construct their corresponding layer

[Step 7] Check the termination criterion

[Step 8] Determine new input variables for the next layer

\section{Experimental Studies}

We demonstrate how the IG-based gFSPNN can be utilized to predict future values of a chaotic Mackey-Glass time series. This time series is used as a benchmark in fuzzy 
Table 2. Summary of the parameters of the genetic optimization

\begin{tabular}{|c|c|c|c|}
\hline & Parameters & $1^{\text {st }}$ layer & $2^{\text {nd }}$ to $3^{\text {rd }}$ layer \\
\hline \multirow{6}{*}{ GA } & Maximum generation & 150 & 150 \\
\hline & Total population size & 300 & 300 \\
\hline & Selected population size $(W)$ & 30 & 30 \\
\hline & Crossover rate & 0.65 & 0.65 \\
\hline & Mutation rate & 0.1 & 0.1 \\
\hline & String length & $\operatorname{Max} * 2+1$ & $\operatorname{Max} * 2+1$ \\
\hline \multirow{7}{*}{ FSPNN } & $\begin{array}{l}\text { Maximal no.(Max) of inputs to be } \\
\text { selected }\end{array}$ & $1 \leq l \leq \operatorname{Max}(2 \sim 5)$ & $1 \leq l \leq \operatorname{Max}(2 \sim 5)$ \\
\hline & $\begin{array}{l}\text { Polynomial type (Type } \mathrm{T} \text { ) of the } \\
\text { consequent part of fuzzy rules }\end{array}$ & $1 \leq \mathrm{T} \leq 4$ & $1 \leq \mathrm{T} \leq 4$ \\
\hline & \multirow{2}{*}{$\begin{array}{c}\text { Consequent input type to be used } \\
\text { for Type } \mathrm{T}(*)\end{array}$} & Type T & Type T \\
\hline & & Type $\mathrm{T}^{*}$ & Type T \\
\hline & \multirow{2}{*}{ Membership Function (MF) type } & Triangular & Triangular \\
\hline & & Gaussian & Gaussian \\
\hline & No. of MFs per input & $2 \sim 5$ & $2 \sim 5$ \\
\hline
\end{tabular}

$l$, T, Max: integers, $\mathrm{T}^{*}$ means that entire system inputs are used for the polynomial in the conclusion part of the rules.

and neurofuzzy modeling. The time series is generated by the chaotic Mackey-Glass differential delay equation. To come up with a quantitative evaluation of the network, we use the standard RMSE performance index. Table 2. depicts the parameters of genetic optimization related to the proposed network.

The population size being selected from the total population size (300) is equal to 30 . The process is realized as follows. 150 nodes are generated in each layer of the network. The parameters of all nodes generated in each layer are estimated and the network is evaluated using both the training and testing data sets. Then we compare these values and choose 30 FSPNs that produce the best (lowest) value of the performance index. The maximal number (Max) of inputs to be selected is confined to two to five (2-5) entries. The polynomial order of the consequent part of fuzzy rules is chosen from four types, that is Type 1, Type2, Type 3, and Type 4.

Fig. 1 depicts the performance index of each layer of gFSPNN according to the increase of maximal number of inputs to be selected. In Fig. $1, \mathrm{~A}(\bullet)-\mathrm{D}(\bullet)$ denote the optimal node numbers at each layer of the network.

Fig. 2 illustrates the detailed optimal topologies of gFSPNN for three layer when using Max=5. As shown in Fig 2, the proposed network enables the architecture to be a structurally more optimized and simplified network than the conventional FPNN. In nodes (FSPNs) of Fig. 2, 'FSPNn' denotes the $n^{\text {th }}$ FSPN (node) of the corresponding layer, the number of the left side denotes the number of nodes (inputs or FSPNs) 


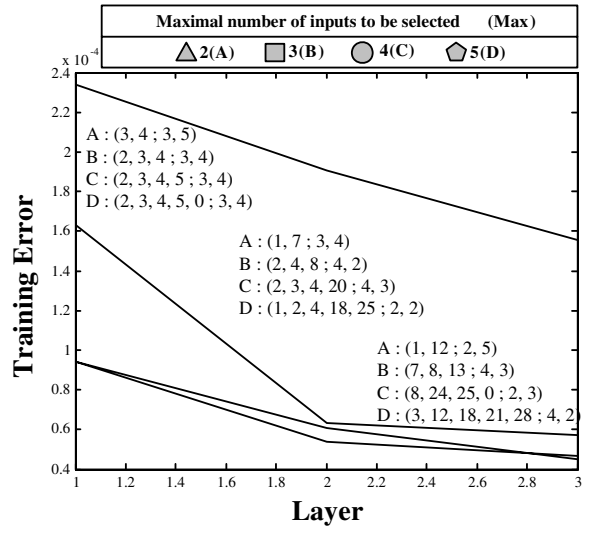

(a-1) Training error

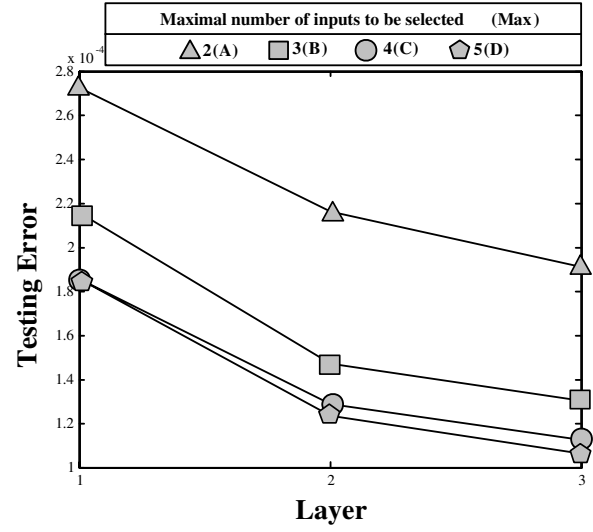

(a-2) Testing error

(a) Triangular membership function

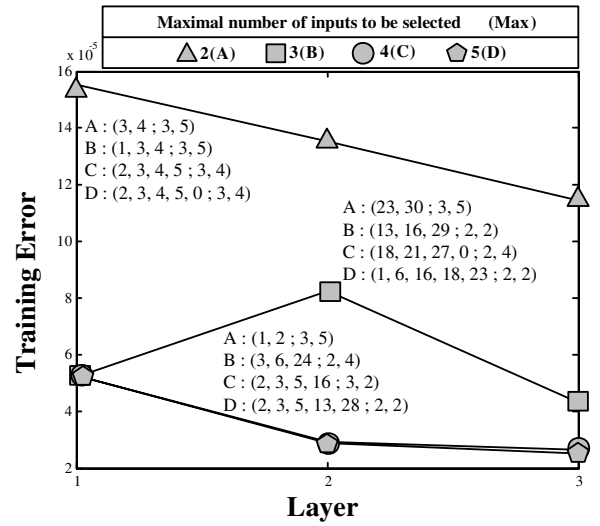

(b-1) Training error

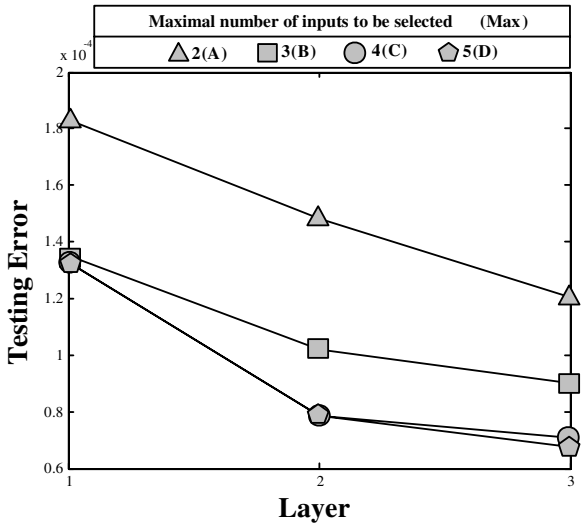

(b-2) Testing error

(b) Gaussian-like membership function

Fig. 1. Performance index of IG-gFSPNN (with Type $T^{*}$ ) with respect to the increase of number of layers

coming to the corresponding node, the number of the center denote the polynomial order of conclusion part of fuzzy rules used in the corresponding node and the number of the right side denotes the number of membership functions.

Table 3 summarizes a comparative analysis of the performance of the network with other models. The experimental results clearly reveal that it outperforms the existing models both in terms of significant approximation capabilities (lower values of the performance index on the training data, $\mathrm{PI}_{\mathrm{s}}$ ) as well as superb generalization abilities (expressed by the performance index on the testing data $\mathrm{EPI}_{\mathrm{s}}$ ). The proposed IG based genetically optimized FSPNN (IG-based gFSPNN) can be much lower in comparison to the conventional optimized FPNN as shown in Table 3. 


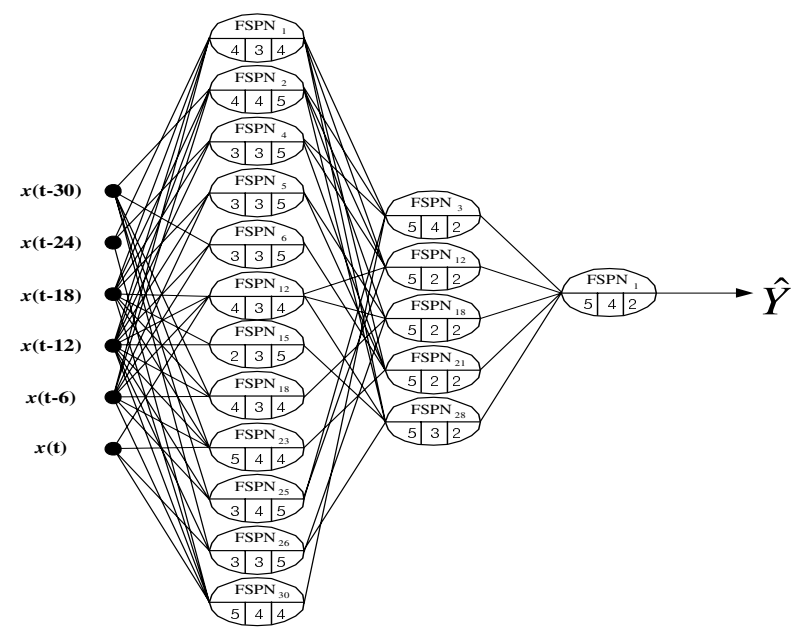

Fig. 2. Optimal networks structure of GAs-based FPNN ( for 3 layers )

Table 3. Comparative analysis of the performance of the network; considered are models reported in the literature

\begin{tabular}{|c|c|c|c|c|c|c|}
\hline \multirow{2}{*}{\multicolumn{4}{|c|}{ Model }} & \multicolumn{3}{|c|}{ Performance index } \\
\hline & & & & PI & $\mathrm{PI}_{\mathrm{s}}$ & $\mathrm{EPI}_{\mathrm{s}}$ \\
\hline \multirow{3}{*}{\multicolumn{4}{|c|}{ Wang's model[9] }} & 0.044 & & \\
\hline & & & & 0.013 & & \\
\hline & & & & 0.010 & & \\
\hline \multicolumn{4}{|c|}{ ANFIS[10] } & & 0.0016 & 0.0015 \\
\hline \multicolumn{4}{|c|}{ FNN model[11] } & & 0.014 & 0.009 \\
\hline \multirow{2}{*}{$\begin{array}{c}\text { Proposed } \\
\text { IG- } \\
\text { gFSPNN }\end{array}$} & \multirow{2}{*}{ Type T* } & $\begin{array}{l}\text { Triangular } \\
\left(3^{\text {nd }} \text { layer }\right)\end{array}$ & $\operatorname{Max}=5$ & & $4.65 e-5$ & $1.06 \mathrm{e}-4$ \\
\hline & & $\begin{array}{c}\text { Gaussian } \\
\left(3^{\text {nd }} \text { layer }\right)\end{array}$ & Max $=5$ & & $2.49 \mathrm{e}-5$ & $6.76 e-5$ \\
\hline
\end{tabular}

\section{Concluding Remarks}

In this study, we have surveyed the new structure and meaning of fuzzy rules and investigated the GA-based design procedure of Fuzzy Polynomial Neural Networks (FPNN) along with its architectural considerations. The whole system is divided into some sub-systems that are classified according to the characteristics named information granules. Each information granule seems to be a representative of the related sub-systems. A new fuzzy rule with information granule describes a sub-system as a stand-alone system. A fuzzy system with some new fuzzy rules depicts the whole system as a combination of some stand-alone sub-system.

The GA-based design procedure applied at each stage (layer) of the FSPNN leads to the selection of the preferred nodes (or FSPNs) with optimal local characteristics (such as the number of input variables, the order of the consequent polynomial of fuzzy rules, and input variables) available within FSPNN. The comprehensive 
experimental studies involving well-known datasets quantify a superb performance of the network in comparison to the existing fuzzy and neuro-fuzzy models.

Acknowledgements. This work was supported by the Korea Research Foundation Grant funded by the Korean Government (MOEHRD)(KRF-2006-311-D00194, Basic Research Promotion Fund).

\section{References}

1. Pedrycz, W.: Computational Intelligence: An Introduction, CRC Press, Florida, (1998)

2. Peters, J.F, Pedrycz, W.: Computational intelligence, In Encyclopedia of Electrical and Electronic Engineering, (Edited by J.G. Webster). John Wiley \& Sons, New York. 22 (1999)

3. Pedrycz, W., Peters, J.F. (ed.): Computational Intelligence in Software Engineering, World Scientific, Singapore. (1998)

4. Takagi, H., Hayashi, I.: NN-driven fuzzy reasoning, Int. J. of Approximate Reasoning. 5 (3) (1991), 191-212

5. S.-K. Oh and W. Pedrycz, "Self-organizing Polynomial Neural Networks Based on PNs or FPNs : Analysis and Design", Fuzzy Sets and Systems, 2003(in press).

6. Z. Michalewicz, "Genetic Algorithms + Data Structures = Evolution Programs", Springer-Verlag, Berlin Heidelberg, 1996.

7. D. Jong, K. A., “Are Genetic Algorithms Function Optimizers?”, Parallel Problem Solving from Nature 2, Manner, R. and Manderick, B. eds., North-Holland, Amsterdam.

8. S.-K. Oh and W. Pedrycz, "Fuzzy Polynomial Neuron-Based Self-Organizing Neural Networks”, Int. J. of General Systems, Vol. 32, No. 3, pp. 237-250, May, 2003.

9. L. X. Wang, J. M. Mendel, "Generating fuzzy rules from numerical data with applications", IEEE Trans. Systems, Man, Cybern., Vol. 22, No. 6, pp. 1414-1427, 1992.

10. J. S. R. Jang, “ANFIS: Adaptive-Network-Based Fuzzy Inference System”, IEEE Trans. System, Man, and Cybern., Vol. 23, No. 3, pp. 665-685, 1993.

11. L. P. Maguire, B. Roche, T. M. McGinnity, L. J. McDaid, "Predicting a chaotic time series using a fuzzy neural network", Information Sciences, Vol. 112, pp. 125-136, 1998.

12. S.-K. Oh, W. Pedrycz and T.-C. Ahn, "Self-organizing neural networks with fuzzy polynomial neurons", Applied Soft Computing, Vol. 2, Issue 1F, pp. 1-10, Aug. 2002.

13. L. A. Zadeh, "Toward a theory of fuzzy information granulation and its centrality in human reasoning and fuzzy logic", Fuzzy Sets Syst, vol. 90, pp. 111-117, 1997

14. Park, B.J., Lee, D.Y., Oh, S.K.: Rule-based Fuzzy Polynomial Neural Networks in Modeling Software Process Data. Int. J. of Control Automation and Systems. 1(3) (2003) 321-331 